BNL-73616-2005-CP

\title{
Atomic Beam Studies in the RHIC H-Jet Polarimeter
}

\author{
A. Zelenski, D. Graham, S. Kokhanovski, G. Mahler, Y. Makdisi, A. Nass, J. \\ Ritter, and V. Zubets (BNL) \\ W. Haeberli and T. Wise (U. of Wisconsin-Madison)
}

Presented at $16^{\text {th }}$ International SPIN Physics Symposium

Trieste, Italy, October 10-16, 2004

January 2005

\author{
Collider-Accelerator Department \\ Brookhaven National Laboratory \\ P.O. Box 5000 \\ Upton, NY 11973-5000 \\ www.bnl.gov \\ Managed by \\ Brookhaven Science Associates, LLC \\ for the United States Department of Energy under \\ Contract No. DE-AC02-98CH10886
}




\section{DISCLAIMER}

This report was prepared as an account of work sponsored by an agency of the United States Government. Neither the United States Government nor any agency thereof, nor any of their employees, nor any of their contractors, subcontractors, or their employees, makes any warranty, express or implied, or assumes any legal liability or responsibility for the accuracy, completeness, or any third party's use or the results of such use of any information, apparatus, product, or process disclosed, or represents that its use would not infringe privately owned rights. Reference herein to any specific commercial product, process, or service by trade name, trademark, manufacturer, or otherwise, does not necessarily constitute or imply its endorsement, recommendation, or favoring by the United States Government or any agency thereof or its contractors or subcontractors. The views and opinions of authors expressed herein do not necessarily state or reflect those of the United States Government or any agency thereof. 


\title{
ATOMIC BEAM STUDIES IN THE RHIC H-JET POLARIMETER
}

\author{
A. ZELENSKI, D. GRAHAM, S. KOKHANOVSKI, G. MAHLER, Y. \\ MAKDISI, A. NASS, J. RITTER AND V. ZUBETS \\ Brookhaven National Laboratory, Bldg. 930 \\ Upton, $N Y$ 11973, USA \\ W. HAEBERLT AND T. WISE \\ Department of Physics, University of Wisconsin-Madison \\ Madison, Wisconsin 53706, USA
}

\begin{abstract}
The results of atomic beam production studies are presented. Improved cooling of the atoms before jet formation in the dissociator cold nozzle apparently reduces the atomic beam velocity spread and improves beam focusing conditions. A carefully designed sextupole separating (and focusing) magnet system takes advantage of the high brightness source. As a result a record beam intensity of a $12.4 \cdot 10^{16}$ atoms/s was obtained within $10 \mathrm{~mm}$ acceptance at the collision point. The results of the polarization dilution factor measurements (by the hydrogen molecules at the collision point) are also presented.
\end{abstract}

A polarized $\mathrm{H}$-jet polarimeter is based on elastic proton-proton scattering in the Coulomb-Nuclear Interference (CNI) region. Due to particle identity, the polarization of the accelerated proton beam can be directly expressed in terms of proton target polarization, which can be precisely measured by a Breit-Rabi polarimeter. A detailed H-jet polarimeter description and results of the beam intensity and density measurements were presented at the PST 2003 in Novosibirsk ${ }^{1}$. Atomic polarization measurements and the $\mathrm{H}$-jet project overview are presented at this conference (A. Nass et al. and T. Wise et al. at this conference). The focus of this paper is atomic beam production, dissociator long-term performance and the first measurements of polarization dilution factor by molecular hydrogen.

Dissociator. In a conventional dissociator design hydrogen atoms are produced in an RF-discharge with velocities equivalent to a few thousand degrees gas temperatures. The atoms are cooled down in collisions with the pyrex or quartz walls of the dissociator discharge tube. The tube is usually 
cooled by a water jacket and only the aluminum nozzle at the end of the tube is cooled to $30-100 \mathrm{~K}$ temperatures. As was described in ${ }^{1}$, a new dissociator design has an extended $12 \mathrm{~cm}$ cold tube section upstream of the nozzle is cooled by cryocooler to $70-140 \mathrm{~K}$. This allows a reduction in wall surface recombination, improves hydrogen atoms thermal equilibrium conditions, and reduces the beam velocity spread.

A number of different dissociator "neck" and nozzle cooling system modification were tested over the last year. These cooling improvements resulted in beam intensity increases for the same nozzle temperature. The cooling improvement allows the dissociator operation at higher RF-power. The operational power was increased to $250 \mathrm{~W}$. At higher power $280-300 \mathrm{~W}$ the beam intensity is about $10 \%$ higher but the nozzle cooling is insufficient. At this power the RF-discharge penetrates further in the cold "neck" where wall recombination is reduced at $80-140 \mathrm{~K}$ temperatures. Also an ice layer formation at the tube and nozzle surfaces reduces recombination. There is a significant heat transfer to the heat sink copper clamp (attached to the cryocooler) from plasma cooling and recombination. This heat transfer is varying in time for the same input RF-power and depends on the tube wall conditions and the quality of the thermal contact between the copper clamp and the quartz tube. The better contact actually reduces the heat load due to a decrease in the tube wall temperature, which reduces recombination. The copper clamp-quartz tube thermal conductance is limited in all of our tested designs. At present a thin $(\sim 0.2 \mathrm{~mm})$ layer of "Apiezon" cryogenic vacuum grease (instead of finger springs ${ }^{1}$ ) is used to provide the heat conductance between copper and quartz. It worked quite reliably during the RHIC run, but the reproducibility of the grease application is still a problem and needs to be improved.

A production of silicon oxide in discharge plasma reaction with the quartz tube sometimes causes the nozzle plugging by a "snow-ball" of $\mathrm{SiO}_{2}$. The oxide is accumulated in the region of plasma boundary. The plasma penetrates about halfway in the cold dissociator "neck" and the wall temperature at this point is about $130 \mathrm{~K}$. At continuous operation the oxide is bounded perhaps by ice and a stable dissociator operation up to 3 weeks was recorded. But sometimes the "snow-ball" plugged the nozzle completely just after a 5 -day operation. The procedure of vacuum cleaning of the dissociator with the plastic tube attached to the vacuum pump was developed and effectively used during the H-jet run in the RHIC. Less than 0.5-hour ring access was required to fulfill this procedure.

In the H-jet dissociator the cold surface area of the "neck" and nozzle 

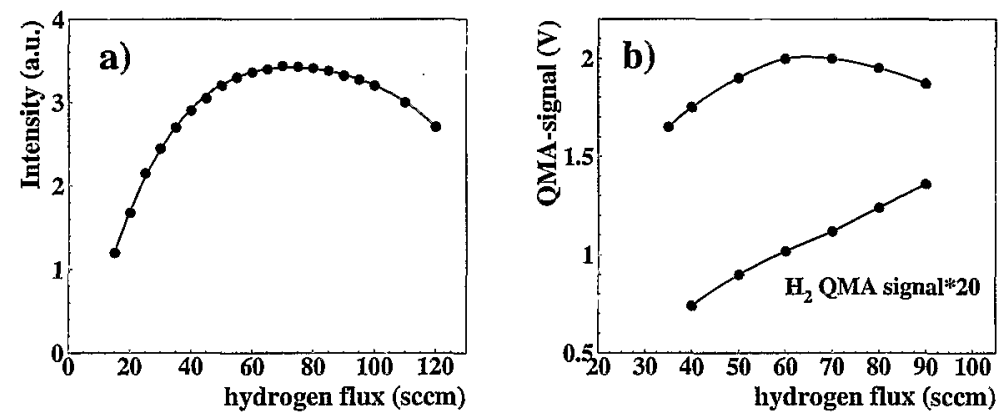

Figure 1. Atomic beam intensity vs. $\mathrm{H}_{2}$ flow in dissociator (a). Atomic and molecular hydrogen density vs. $\mathrm{H}_{2}$ flow as measured by the QMA (b).

is much larger than in the conventional dissociator, where just the nozzle is cold. The water vapor which is produced mostly in the discharge condensed and froze upstream of the nozzle and there is no a need to warm the dissociator up for the nozzle cleaning from ice. During three weeks of continuous operation there was observed a steady increase (about 10\%) in the beam intensity. This effect can be explained by a reduction in recombination due to better wall coating by the ice.

Atomic Beam Intensity Measurements. The total beam intensity in the collision chamber was measured with a compression tube $10 \mathrm{~mm}$ in diameter and $100 \mathrm{~mm}$ length. The compression tube was calibrated by the conventional technique ${ }^{1}$. The maximum atomic beam intensity of a $(12.4 \pm 0.2) \cdot 10^{16}$ atoms $/ \mathrm{sec}$ was measured at $\sim 75 \mathrm{sccm}$ flow, the dissociator RF-power was $280 \mathrm{~W}$ and nozzle temperature of $75 \mathrm{~K}$ (see Fig.1a). The maximum intensity is significantly higher than in other ABS. Remarkably the HERMES ABS intensity of a $6.4 \cdot 10^{16}$ atoms/s was obtained at just $30 \mathrm{sccm} \mathrm{H}_{2}$ flow i.e. three time less gas supply in dissociator, than in the HERMES $\mathrm{ABS}^{2}$. This intensity dependence was measured after the dissociator cooling upgrade. The intensity decrease rate is not as fast as before ${ }^{1}$ and the maximum is shifted to the higher flow. Apparently precooling the atoms before the nozzle is essential for the beam formation out of the nozzle and insufficient cooling limits the intensity increase. At higher $\mathrm{H}_{2}$ flow the heat load to the dissociator "neck" is increased and plasma penetrates further into the "neck", this may change the equilibrium velocity distribution and affect the beam quality. The dissociation ratio might also decrease. 
The operational temperature of the cooling bracket in the top part of the "neck" is about $140 \mathrm{~K}$, which is close to minimum for recombination reduction at the quartz. But the inside wall temperature can be significantly higher. The cooling system improvement is still required.

The target polarization dilution by molecular hydrogen.

Atomic beam polarization close to $96 \%$ was measured in the Breit-Rabi polarimeter (A. Nass et al. at this conference). The target polarization dilution by the molecular hydrogen (at the collision point) was measured by a modified quadrupole mass analyzer. The original RIBEN QMA sensitive area was expanded from $5 \mathrm{~mm}$ to $10 \mathrm{~mm}$ to accommodate the full beam profile of a $9 \mathrm{~mm}$ (at the base) wide. This significantly reduced the atomic beam recombination at the QMA electrodes, which was observed in the first measurements. The QMA signal amplitude dependence on hydrogen flow in the dissociator is presented in Fig.1b. The maximum of QMA atomic beam signal is shifted to a lower flow from the compression tube maximum. In difference from compression tube, the QMA measures the beam density not intensity. The density is a figure of merit for the H-jet target. Therefore the maximum of the QMA signal (at $60 \mathrm{sccm}$ flow) is the best operational point. The absolute atomic beam density of about $1.0 \cdot 10^{12}$ atoms $/ \mathrm{cm}^{3}$ at the collision point can be calculated from the atomic beam intensity, the beam profile and velocity. The QMA sensitivity factor to the molecular hydrogen the QMA signal was calibrated at different hydrogen pressures (hydrogen gas was supplied to the collision chamber through a needle valve) measured by a hot filament ion gauge closely positioned to the QMA sensor. The molecular hydrogen density of about $1.5 \%$ of atomic beam density was determined from these measurements, which provides a preliminary measurement of the polarization dilution factor of about $3 \%$ at $60 \mathrm{sccm} \mathrm{H}_{2}$ flow in dissociator. This gives an effective $\mathrm{H}$-jet target polarization of a $93_{-2}^{+1} \%$. To meet the project goal of a $1 \%$ absolute accuracy for $\mathrm{H}$-jet target polarization a new diagnostics devices are under development. A device for off-line measurements is based on electron beam ionizer and magnetic analysis for ion components. For on-line polarization dilution monitoring an optical technique development is in progress. It is based on detection of a luminescence light from atoms and molecules exited by the RHIC proton beam.

\section{References}

1. A. Zelenski et al., Proc. PST 2003, NIM A 26367 (2004).

2. A. Nass et al., Nucl. Inst. and Meth. A505 (2003), 633. 